\title{
Interaction of hydro-elastic waves with a vertical wall
}

\author{
Paul Brocklehurst, Alexander Korobkin and Emilian Parau \\ Department of Mathematics \\ University of East Anglia \\ Norwich, England
}

January 13, 2010

\section{Introduction}

This study aims to gain knowledge on how hydroelastic waves interact with off-shore structures, for example off-shore wind turbines or oil rigs. The linear two-dimensional problem of hydroelastic waves reflected by a vertical wall is analysed. Water is of finite depth and is covered by ice. The fluid is assumed incompressible and inviscid. The ice sheet is assumed thin compared with both the water depth and length of the incident wave. Deflection of the ice sheet is described by linear elastic plate theory (see for example [1]), and fluid flow by using the potential flow model [5]. The ice sheet extends infinitely and is clamped to the verticalwalled structure. The incident hydroelastic wave is regular. The explicit solution for the ice deflection is derived, as well as the solution for the velocity potential in the fluid. We calculate several quantities of practical importance for off-shore structures in ice-covered waters, for instance the vertical shear force. The clamped ice introduces the vertical force acting on the structure in contrast to the more familiar problem of ice free floating on the water surface, where such force is absent. The vertical force is periodic in time and should be taken into account in the pre-design stage for such a structure. The magnitude of the vertical force is of the same order as that of the horizontal wave force component, which also is calculated. The horizontal force is analysed in detail to determine which terms in the model make the most contribution. Comparison is also made to the horizontal force of regular flexural gravity waves with no ice sheet present. The pressure on the wall and the bending stresses in the ice plate are also calculated.

\section{Mathematical formulation}

The geometry of the problem and co-ordinate system are shown in figure (1). We introduce Cartesian coordinates with the $x$-axis being the bottom of the ice sheet at rest and the $y$-axis directed vertically upwards. Time is denoted by $t$. The ice sheet is clamped to the wall at the origin. The fluid bed is flat and the fluid has depth $H$. The pressure in the fluid is represented by $p(x, y, t)$, and the density of the fluid by $\rho$. The fluid velocity $\mathbf{V}(x, y, t)$ is equal to the gradient of the velocity potential $\phi(x, y, t)$, hence $\nabla \phi=\mathbf{V}$.

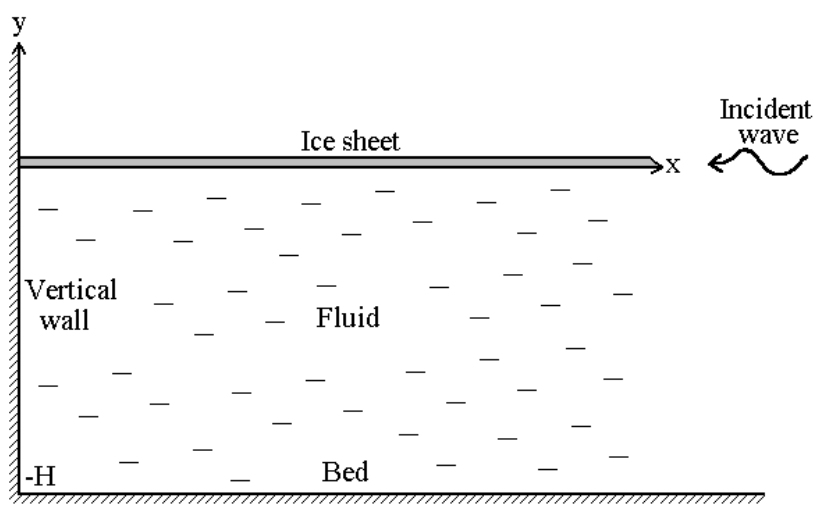

Figure 1: Schematic of an infinite ice sheet meeting a vertical wall. 
The deflection of the ice sheet is denoted by $w(x, t)$. The ice has mass per unit length $M$, where $M=\rho_{i} h$. Here $\rho_{i}$ is the ice density and $h$ is the ice thickness. The incident wave parameters are: $a$, wave amplitude; $\omega$, wave frequency; $\theta$, phase shift; $k$, wavenumber and $c$, phase velocity. Typical values of each parameter are taken from measurements in McMurdo Sound in Antarctica [4] and used here as the reference data set. The problem is formulated and studied within the linear theory of hydroelasticity [3]. The velocity potential $\phi(x, y, t)$ satisfies Laplace's equation in the flow region;

$$
\nabla^{2} \phi=0, \quad(-H \leq y \leq 0, x>0) .
$$

The hydrodynamic pressure in the fluid is given within the linear theory as (subscripts indicate partial derivatives)

$$
p=-\rho \phi_{t}-\rho \mathrm{g} y, \quad(-H \leq y \leq 0, x>0) .
$$

Within linear elastic plate theory the differential equation for the ice deflection is given as

$$
E J w_{x x x x}+M w_{t t}=p(x, 0, t), \quad(y=0, x>0),
$$

where $E$ is Young's modulus and $J=h^{3} /\left[12\left(1-\nu^{2}\right)\right]$, where $\nu$ is Poisson's ratio. EJ is known as the 'flexural rigidity' of the elastic plate. Boundary conditions for the Laplace equation (2.1) are

$$
\begin{aligned}
\phi_{x}=0, & (-H \leq y \leq 0, x=0), \\
\phi_{y}=0, & (y=-H, x>0), \\
\phi_{y}=w_{t}(x, t), & (y=0, x>0) .
\end{aligned}
$$

The boundary conditions (2.4)-(2.6) are imposed on the vertical wall, the bottom and the ice sheet respectively. The edge conditions for the ice plate on the wall are

$$
w=0, w_{x}=0, \quad(x=0) .
$$

These conditions imply that the ice plate is clamped to the wall at the origin. In the far field, $x \rightarrow \infty$, the velocity potential $\phi(x, y, t)$ and plate deflection $w(x, t)$ consist of two components. The first terms represent the incident hydroelastic wave with amplitude $a$ and frequency $\omega$. The second terms represent the wave propagating from the wall with complex amplitude $a_{D}$ which is unknown and must be determined as part of the solution. There are no initial conditions in this formulation because we are seeking a time-periodic solution of the form [2]

$$
\begin{aligned}
\phi(x, y, t) & =\operatorname{Re}\left(\Phi(x, y) \mathrm{e}^{-\mathrm{i} \omega t}\right), \\
w(x, t) & =\operatorname{Re}\left(\frac{\mathrm{i}}{\omega} W(x) \mathrm{e}^{-\mathrm{i} \omega t}\right),
\end{aligned}
$$

where the complex valued functions $\Phi(x, y)$ and $W(x)$ are unknown.

A solution to the problem (2.1)-(2.7) is sought in the following form:

$$
\begin{aligned}
\Phi(x, y) & =\frac{a \omega}{k} \frac{\cosh (k[y+H])}{\sinh (k H)} \mathrm{e}^{-\mathrm{i} k x}+\frac{a_{D} \omega}{k} \frac{\cosh (k[y+H])}{\sinh (k H)} \mathrm{e}^{\mathrm{i} k x}+\varphi(x, y), \\
W(x) & =a \omega \mathrm{e}^{-\mathrm{i} k x}+a_{D} \omega \mathrm{e}^{\mathrm{i} k x}+\hat{w}(x),
\end{aligned}
$$

where $a_{D}$ is the complex amplitude of the reflected wave. In (2.10) and (2.11), terms on the right hand sides correspond respectively to the incident waves, reflected waves and unknown functions $\varphi(x, y)$ and $\hat{w}(x)$ to be determined. The far field condition implies that $\hat{w}$ and $\varphi$ decay far from the wall. The problem can then be reformulated in terms of the unknown functions $\hat{w}(x)$ and $\varphi(x, y)$.

\section{Method of Solution}

To solve the problem we apply a Fourier cosine transform:

$$
\begin{aligned}
\varphi^{c}(\xi, y) & =\int_{0}^{\infty} \varphi(x, y) \cos (\xi x) \mathrm{dx}, \\
w^{c}(\xi) & =\int_{0}^{\infty} \hat{w}(x) \cos (\xi x) \mathrm{dx} .
\end{aligned}
$$


The conditions at infinity allow us to repetitively apply integration by parts to Laplace's equation (2.1) and the plate equation (2.3) and obtain a solution. We find that

$$
\varphi^{c}(\xi, y)=\frac{\cosh (\xi[y+H])}{\xi \sinh (\xi H)}\left(w^{c}(\xi)-\frac{\mathrm{i} k \omega\left(a-a_{D}\right)}{k^{2}-\xi^{2}}\right)+\frac{S}{k^{2}-\xi^{2}} \cosh (k[y+H]),
$$

where

$$
S=\omega \mathrm{i} \frac{a-a_{D}}{\sinh (k H)}
$$

After considerable work on the plate equation (2.3) we find that

$$
w^{c}(\xi)=\mathrm{i} \rho \omega^{3}\left(a-a_{D}\right) Q(\xi),
$$

where

$$
Q(\xi)=\frac{\xi \tanh (\xi H)\left(V(k H)+E J \frac{k}{\rho \omega^{2}}\left(k^{2}-\xi^{2}\right)\right)+\frac{1}{k^{2}-\xi^{2}}\left(\frac{\xi \tanh (\xi H)}{\tanh (k H)}-k\right)}{\xi \tanh (\xi H)\left(E J \xi^{4}+\rho \mathrm{g}-M \omega^{2}\right)-\rho \omega^{2}},
$$

and

$$
V(k H)=\frac{1}{2 k^{2} \tanh (k H)}+\frac{H}{2 k \sinh ^{2}(k H)} .
$$

With the fluid depth used as a length scale, the problem is rewritten in dimensionless variables, and dimensionless versions of $w^{c}(\xi), \varphi^{c}(\xi)$ and the important function $Q(\xi)$ are obtained. With this nonimensionalisation the problem is reduced to three dimensionless parameters; $k^{*}, \xi^{*}$ and $\gamma^{*}$, with $\gamma^{*}=E J /\left(\rho \omega^{2} H^{5}\right)$. The function $Q(\xi)$ is analysed in detail, particularly its behaviour as $\xi \rightarrow k$. Here, the denominator is zero, but the numerator is also defined to be zero hence avoiding a singularity. With $\varphi^{c}(\xi, y)$ and $w^{c}(\xi)$ obtained, we can perform inverse Fourier cosine transforms which are too complex to be performed analytically. However, the integrals involved converge reasonably fast and we can calculate the required distributions numerically. It is possible to use the remaining boundary condition (2.7) to obtain

$$
a_{D}=a \mathrm{e}^{\mathrm{i} \theta},
$$

where $\theta$ is the phase shift between the incident and reflected waves. The phase shift $\theta$ has been studied and is calculated by quadratures and the characteristics of the ice and incident waves.

\section{Numerical results}

A sample of numerical results is presented in figure (2). We are mostly interested in varying the wavenumber $k^{*}$ to investigate how this effects various results, and other parameters such as the fluid depth and ice thickness are also varied. When dealing with waves in ice, we are mostly interested in long waves in accordance with the linear theory. Waves with periods of $2 \mathrm{~s}-25 \mathrm{~s}$ correspond to wavenumbers $k^{*}=1-30$. Within figure (2), (a) shows the function $Q^{*}\left(\xi^{*}\right)$ plotted against $\xi^{*} . Q^{*}$ represents the contribution to the deflection caused by the ice clamping condition, and hence is paramount in the solution. $Q^{*}$ decays as $\xi \rightarrow \infty$, as required by the far field conditions. Figure (b) shows the first three wave peaks of the ice sheet deflection. We see that the deflection is zero at the origin, as is its slope, as required by the ice clamping conditions. The deflection is smallest in the first wave peak due to this clamping. Here the fluid depth is also varied to indicate how varying $\gamma$ has a significant effect on the ice deflection. We have,$- H=50 \mathrm{~m}$; ,$--- H=100 \mathrm{~m} ; \cdots \cdots, H=200 \mathrm{~m}$. Figure (c) shows the vertical shear force acting on the wall caused by the flexure of the ice sheet. Figure (d) shows the horizontal wave force component on the wall (without the hydrostatic component). At the workshop this force will be analysed in detail and also compared to free surface waves.

The yield strength of a material is defined as the strain at which a material begins to deform plastically. We do not deal with any cracking or plastic deformation of ice in this study, and hence we only consider conditions that lead to the strains in the ice sheet being below yield. The simplest way to achieve this is to scale the incident wave amplitude to ensure the wave slope is small enough to keep the strain below yield. The strain in the ice sheet is calculated via the second spatial derivative of the deflection $w(x, t)$ (which requires extra attention), and will be presented at the workshop along with the amplitude scale. 
(a)

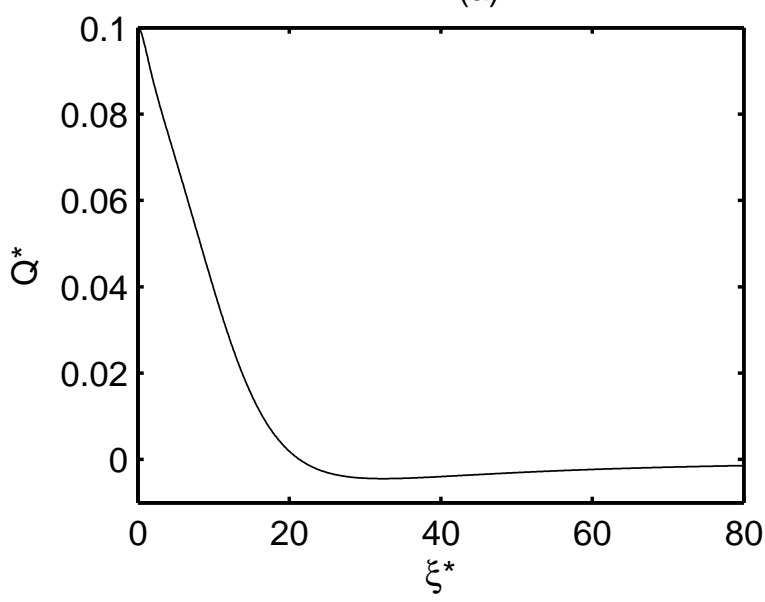

(c)

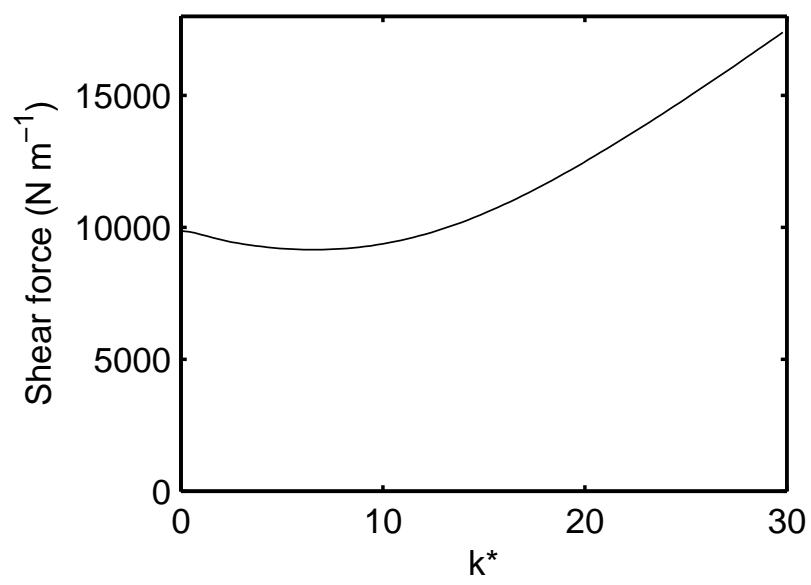

(b)

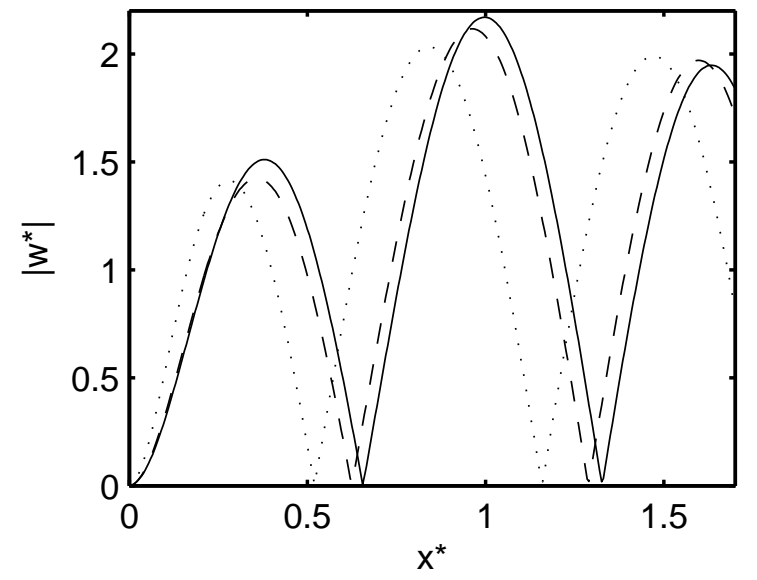

(d)

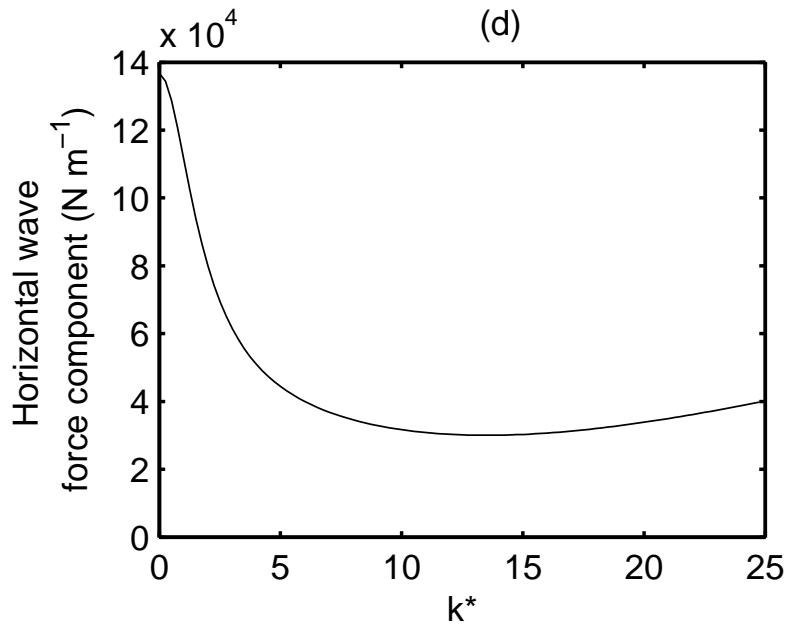

Figure 2: Various numerical results.

\section{Summary}

The ice clamping condition leads to a specific effect on the ice deflection. Both the vertical shear force and the horizontal wave force are considerable. Physical parameters can be easily varied through the dimensionless parameter $\gamma^{*}$. The strain in the ice sheet is highest at the origin where the ice is clamped to the wall; the strain here is considerably higher than in a regular hydroelastic wave, requiring smaller permitted amplitude. If the ice were to crack or break, it would most likely do so at the origin, and the problem could then be reconsidered with different boundary conditions. Results will be presented in more detail at the workshop, including the strain in the ice sheet and further investigation into the horizontal force. The problem of hydro-elastic waves incident on a vertical cylinder has been formulated, again with the ice-clamping condition, and numerical results are currently being studied. Future work could include variable bottom topography, a non-vertical wall or an array of cylinders, all in the presence of an ice sheet.

\section{References}

[1] Fox, C., And Squire, V. On the oblique reflexion and transmission of ocean wave at shore fast sea ice. Phil. Trans. R. Soc. Lond. A 347 (1994), 185-218.

[2] Squire, V., Dugan, J., Wadhams, P., Rottier, P., And Liu, A. Of ocean waves and ice sheets. Annu. Rev. Fluid Mech. 27 (1995), $115-168$.

[3] Squire, V., Hosking, R., Kerr, A., and Langhorne, P. Moving Loads on Ice Plates. Kluwer Academic Publishers, 1996.

[4] Squire, V., Robinson, W., Langhorne, P., and Haskell, T. Vehicles and aircraft on floating ice. Nature 333 (1988), $159-161$.

[5] Stoker, J. Water Waves. Interscience Publishers Inc., 1957. 